\title{
Prothrombotic state in patients with stable COPD: an observational study
}

\author{
Christos Kyriakopoulos ${ }^{1}$, Christos Chronis ${ }^{1}$, Evaggelia Papapetrou ${ }^{2}$, Athina Tatsioni ${ }^{3}$, \\ Konstantina Gartzonika ${ }^{4}$, Christina Tsaousi ${ }^{2}$, Athena Gogali ${ }^{1}$, Christos Katsanos ${ }^{1}$, Aikaterini Vaggeli ${ }^{1}$, \\ Charikleia Tselepi ${ }^{1}$, Georgios Daskalopoulos ${ }^{1}$, Stavros Konstantopoulos ${ }^{1}$, Konstantinos Kostikas (i) ${ }^{1}$ and \\ Athanasios Konstantinidis (D) $^{1}$
}

${ }^{1}$ Respiratory Medicine Dept, University Hospital of loannina, Ioannina, Greece. ${ }^{2}$ University Hospital of loannina, Hematology Laboratory, Ioannina, Greece. ${ }^{3}$ Research Unit for General Medicine and Primary Health Care, University of Ioannina, Ioannina, Greece. ${ }^{4}$ Microbiology Dept, University Hospital of Ioannina, Ioannina, Greece.

Corresponding author: Athanasios Konstantinidis (akonstan@uoi.gr)

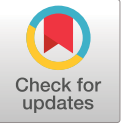

This version is distributed under the terms of the Creative Commons Attribution Non-Commercial Licence 4.0. For commercial reproduction rights and permissions contact permissions@ersnet.org

This article has supplementary material available from openres.ersjournals.com

Editorial comment in ERJ Open Res 2021; 7: 00535-2021

[https://doi.org/10.1183/

23120541.00535-2021].

Received: 29 April 2021 Accepted: 28 July 2021

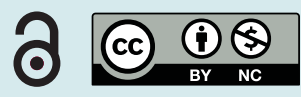

Shareable abstract (@ERSpublications)

Patients with stable COPD exhibit increased levels of key coagulation factors and decreased levels of coagulation inhibitors, namely protein S and antithrombin, compared to COPD-free smokers, indicating a prothrombotic state in stable COPD https://bit.ly/2VmR1PP

Cite this article as: Kyriakopoulos C, Chronis C, Papapetrou E, et al. Prothrombotic state in patients with stable COPD: an observational study. ERJ Open Res 2021; 7: 00297-2021 [DOI: 10.1183/ 23120541.00297-2021].

\section{Abstract}

Background COPD patients have an increased risk of cardiovascular disease and venous thromboembolism.

Methods This study aimed to investigate whether patients with stable COPD have a prothrombotic state compared to COPD-free smokers. We conducted an observational study comparing levels of: D-dimers, INR, aPTT, coagulation factors; fibrinogen, FII, FV, FVII, FVIII, FIX, FX and coagulation inhibitors; protein S, proteins $\mathrm{C}$ and antithrombin between stable COPD patients and control subjects.

Results A total of 103 COPD patients and 42 controls with similar age, sex, current smoking status, comorbidity burden and cardiovascular risk met the inclusion criteria. Compared to controls, COPD patients had higher levels of D-dimers (median (interquartile range): 360 (230-600) ng. $\mathrm{mL}^{-1}$ versus 240 (180-400) $\mathrm{ng} \cdot \mathrm{mL}^{-1}, \mathrm{p}=0.001$ ), fibrinogen (mean $\pm \mathrm{sD}$ : $399 \pm 82 \mathrm{mg} \cdot \mathrm{dL}^{-1}$ versus $346 \pm 65 \mathrm{mg} \cdot \mathrm{dL}^{-1}, \mathrm{p}<0.001$ ), FII $(122 \pm 22 \%$ versus $109 \pm 19 \%$, $\mathrm{p}=0.004), \mathrm{FV}(131 \pm 25 \%$ versus $121 \pm 19 \%, \mathrm{p}=0.015)$, FVIII $(143 \pm 32 \%$ versus $122 \pm 20 \%, \mathrm{p}<0.001)$ and FX $(111(94-134) \%$ versus $98(88-107) \%, \mathrm{p}=0.002)$, and lower levels of protein S (95 (85-105)\% versus $116(98-121) \%, \mathrm{p}<0.001)$ and antithrombin $(94.4 \pm 11.5 \%$ versus 102.3 $\pm 13.2 \%, \mathrm{p}=0.001$ ). In the COPD group, patients with more severe airflow limitation and frequent exacerbations had significantly higher levels of FII, FV and FX, whereas patients with higher COPD assessment test score had significantly higher levels of FX and lower levels of protein S.

Conclusion Patients with stable COPD exhibited increased levels of key coagulation factors and decreased levels of coagulation inhibitors, namely protein S and antithrombin, compared to COPD-free smokers. Among COPD patients, increased levels of FII, FV and FX and decreased levels of protein S were found in patients with more severe disease.

\section{Introduction}

COPD is a chronic debilitating lung disease with a high prevalence of $\sim 380$ million cases worldwide [1]. It is currently the 3rd leading cause of death, responsible for $\sim 6 \%$ of the world's total deaths ( $\sim 3.3$ million annually) [2].

A large body of studies supports the hypothesis that COPD increases the risk for both cardiovascular disease (CVD) and venous thromboembolism (VTE). Thus, COPD patients are more likely to be diagnosed with cardiovascular disease compared with non-COPD subjects, having nearly 2.5 times increased risk of CVD overall, and a two to five times higher risk of major CVD types [3]. In a 
population-based cohort across all stages of COPD during a median follow-up of 6.2 years, subjects with COPD stage III/IV had a two-fold higher risk of secondary VTE compared to subjects with normal airflow [4]. In nested-case-control analyses, in patients with COPD and the same number of COPD-free patients in the UK, the risk of deep vein thrombosis (DVT) (OR 1.35) and pulmonary embolism (OR 2.51) was increased for COPD patients [5]. COPD patients are also at heightened risk of VTE during acute exacerbations of COPD [6], which may be due to clotting system activation [7].

Although the observed association of COPD with CVD and VTE can be partially explained by comorbidities and shared risk factors, there is strong evidence that COPD increases the risk for cardiovascular morbidity and mortality independently of age, sex and smoking history [8, 9]. Mechanisms leading to increased risk of CVD and VTE in COPD include platelet activation [10] and hypercoagulability [11].

A few previous studies have found that stable COPD patients have a prothrombotic phenotype compared to control subjects, manifested by increased levels of circulating tissue factor [12], thrombin-antithrombin complex, fibrinopeptide A, and tissue plasminogen activator inhibitor [13], as well as elevated levels of FII, FVIII, FIX and lower free tissue factor pathway inhibitor [14].

However, there are no previous studies comparing levels of coagulation factors and coagulation inhibitors between patients with stable COPD and COPD-free subjects with similar comorbidity burden and cardiovascular risk. We hypothesised that coagulation factors and inhibitors may differ between patients with stable COPD and those found in COPD-free smokers as well as among COPD patients, and explored potential associations of coagulation factors and coagulation inhibitors with markers of COPD severity, systemic inflammation and lipid profile.

\section{Materials and methods}

This observational study was conducted between December 2017 and November 2019 at the University Hospital of Ioannina, a tertiary Hospital in Greece. 103 consecutive patients with documented COPD were recruited in a respiratory outpatient clinic. 42 volunteers of similar age, sex, comorbidity burden, cardiovascular risk and current smoking status with no history of COPD, confirmed by normal results of standard spirometry, served as control subjects. Control subjects were former or current smokers with $\geqslant 10$-pack-year smoking history and derived from the same outpatient respiratory clinic of our hospital. Those subjects were evaluated for acute respiratory infections excluding pneumonia. They were later contacted and recruited in the study at least 3 months after complete resolution of their symptoms. Control subjects had normal chest radiograph, spirometry and diffusing capacity, and no history or symptoms suggestive of chronic lung disease. The institutional review board of the University Hospital of Ioannina approved this study (IRB No. 28/23-11-2016/20). All study participants provided written informed consent. Results are reported following the Strengthening the Reporting of Observational Studies in Epidemiology (STROBE) guidelines [15].

\section{Patient selection}

Patients were eligible if they were 40 years or more, had $\geqslant 10$-pack-year smoking history and stable disease (defined as the absence of exacerbations or respiratory tract infections within the previous 4 months) with forced expiratory volume in $1 \mathrm{~s}\left(\mathrm{FEV}_{1}\right)$ /forced vital capacity (FVC) ratio $<70 \%$.

The exclusion criteria for both COPD patients and control subjects were: any acute illness, known malignancy, chronic heart failure, chronic renal or liver disease, personal or family history of VTE, previous or current anticoagulant or antiplatelet therapy, history of coronary artery or rheumatic disease. Spirometry was carried out in line with international guidelines (American Thoracic Society (ATS)/ European Respiratory Society (ERS)).

To determine comorbidities, including cardiovascular ones, in all study participants, three data sources were used: 1) self-reported medical history data; 2) data extracted from the electronic medical records of our hospital, which contain all patients' records, including ICD-10 coding and manual data entry, from emergency department, outpatient clinic visits and hospital admissions; and 3) using drug prescription data from the national drug prescription electronic database, which has a full history of all patients' prescribed medications. Data from all three sources were diligently collected and compared, and when a discrepancy occurred, the patient's personal physician was contacted.

\section{Blood collection and analysis}

Fasting blood samples were taken into five tubes with a $4.5-\mathrm{mL}$ volume of $3.2 \%$ trisodium citrate from an antecubital vein with minimal stasis, on the same day that clinical data were recorded. Citrated blood 
samples were centrifuged within $15 \mathrm{~min}$ of collection and stored in aliquots at $-80^{\circ} \mathrm{C}$ until further examination. Biochemical tests were assayed by routine laboratory techniques.

Prothrombin time was determined by clotting assay (STA-Neoplastin R, Diagnostica Stago, Asnieres-surSeine, France) and INR was calculated using mean prothrombin time and the manufacturer's international sensitivity index (ISI). Respectively, activated partial thromboplastin time (aPTT) was measured by clotting assay (STA-Cephascreen, Diagnostica Stago). Clauss method was used for the determination of fibrinogen (STA-Liquid Fib, Diagnostica Stago). The measurement of D-dimer was based on the turbidity method (STA-Liatest D-DI Plus, Diagnostica Stago). Coagulation factor activity for FII, FV, FVII, FVIII, FIX and FX was measured by clotting assay using coagulation factor deficient plasma (Siemens Healthcare Diagnostics, Erlangen, Germany). Protein C and antithrombin were determined by chromogenic activity assay (Berichrom, Siemens Healthcare Diagnostics). Free protein $\mathrm{S}$ antigen was determined by nephelometry (Innovance, Siemens Healthcare Diagnostics). Results for coagulation factors and inhibitors were reported as a percentage of normal. All measurements were performed by a dedicated technician who was blinded to the origin of the samples.

\section{Statistical analysis}

The Kolmogorov-Smirnov and Shapiro-Wilk tests were used to evaluate the normal distribution of data. Categorical values were analysed using the chi-square test or Fisher's exact test as appropriate and were reported as n (\%). Continuous variables were compared by t-test for normally distributed data or by MannWhitney U-test and Kruskal-Wallis test for non-normally distributed data. Continuous parameters with normal distribution are reported as mean $\pm \mathrm{SD}$, while those without normal distribution are reported as median (interquartile range). Univariate regression analyses were performed between each coagulation factor and a group of 11 variables, those related to COPD ( $\mathrm{FEV}_{1}$, diffusing capacity of lung for carbon monoxide (DLCO), COPD assessment test (CAT) score, number of exacerbations in the previous year), and those known to affect coagulation including markers of systemic inflammation (C-reactive protein (CRP), neutrophil count), age, body mass index (BMI), smoking status (introduced as pack-years and current smoking) and low-density lipoprotein (LDL) serum levels. Bonferroni correction was adopted for multiple analyses, and Bonferroni corrected p-value was 0.0045 (0.05/11 variables). Significant factors were subsequently introduced in a multiple linear regression model and controlled for variables known to affect coagulation, including CRP, neutrophil count, age, BMI, smoking status (pack-years and current smoking) and LDL serum levels.

All statistical analyses were performed using SPSS for Windows 26.0 (SPSS, Chicago, IL, USA).

\section{Results}

Characteristics of the participants

From a database of 287 COPD patients who were screened for eligibility, 103 patients (36\%) met the inclusion criteria and 184 (64\%) were excluded. Of 97 COPD-free smoking controls screened, 42 (43\%) met the inclusion criteria (figure 1).

The clinical characteristics, lung function tests and comorbidities are shown in table 1 . The mean age of COPD patients was 63 years; $97 \%$ of the subjects were male, with no differences between the two groups. Cardiovascular risk, as assessed by HEART score, and comorbidities, as assessed by Charlson comorbidity index, were not different between COPD and the control group. BMI was significantly lower in the group of COPD patients $(p=0.015)$. Major comorbidities were arterial hypertension, Type 2 diabetes mellitus and dyslipidaemia, with no difference in their prevalence between the two groups. Proportions of patients classified to ABCD grouping were as follows: Group A, n=28; Group B, n=27; Group C, n=15; Group D, n=33.

\section{Coagulation factors in control subjects and patients with COPD}

Compared with controls, patients with COPD had significantly higher levels of fibrinogen (by 15.3\%, $\mathrm{p}<0.001)$, FII (11.9\%, p=0.004), FV (16.9\%, p=0.015), FVIII $(17.2 \%, \mathrm{p}<0.001)$, FX $(14 \%, p=0.002)$ and D-dimers $(86 \%, \mathrm{p}=0.001)$, and significantly lower protein $\mathrm{S}(4.8 \%, \mathrm{p}<0.001)$ and antithrombin $(7.7 \%$, $\mathrm{p}=0.001$ ) (table 2, figures 2 and 3). FVII, FIX and protein C levels did not differ between the two groups (table 2). When comparisons were performed after excluding participants with diabetes from both groups, the same coagulation factors were significantly different between the two groups (see supplementary table S1).

Levels of coagulation factors in COPD subgroups

In patients with COPD, levels of coagulation factors and inhibitors were determined in subgroups defined by airflow limitation severity, Global Initiative for Chronic Obstructive Lung Disease (GOLD) COPD stage, CAT score and exacerbation history. Significant differences were found predominantly in subgroups 


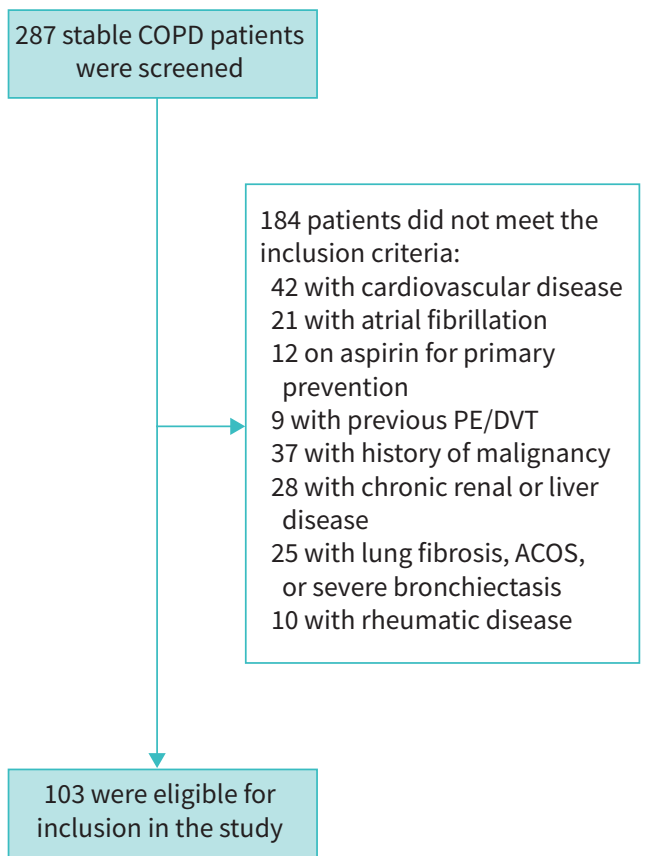

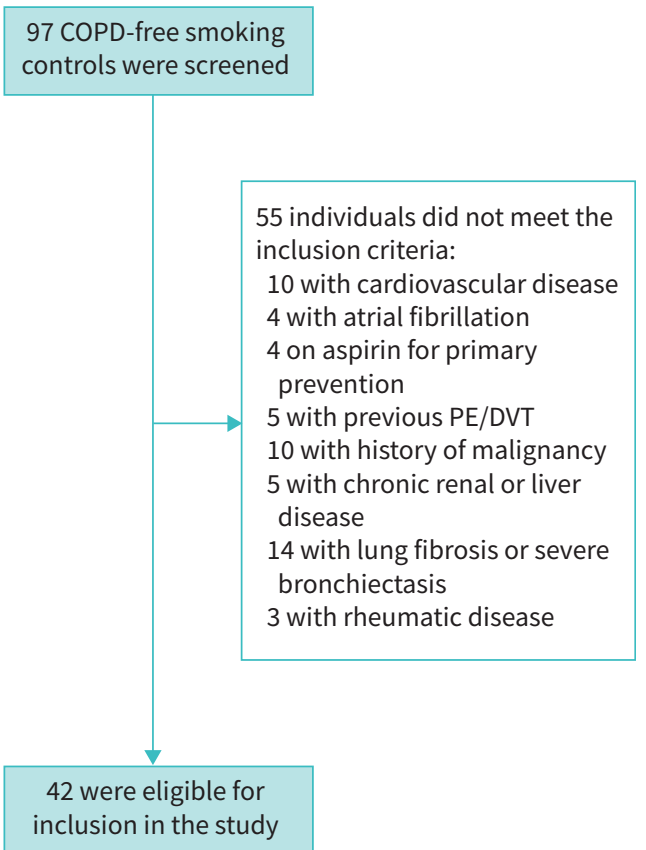

FIGURE 1 Selection flow chart for patients with stable COPD and non-COPD control subjects. PE: pulmonary embolism; DVT: deep vein thrombosis; ACOS: Asthma COPD overlap syndrome.

with more severe disease: 1 ) higher levels of FII in patients with more severe airflow limitation ( $\mathrm{p}=0.003)$, advanced GOLD COPD stage $(p=0.017)$ and frequent exacerbations $(p=0.005)$ (table 3 , figure 4 and supplementary figures S1 and S3); 2) higher levels of FV in patients with more severe airflow limitation $(\mathrm{p}=0.018)$ and frequent exacerbations $(\mathrm{p}=0.018)$ (table 3, figure 4 and supplementary figures S1 and S3); 3) higher levels of FX in patients with more severe airflow limitation ( $p=0.001)$, advanced GOLD COPD stage $(p=0.002)$, frequent exacerbations $(p=0.003)$ and more impaired health status, as reflected by higher CAT score ( $p=0.034$ ) (table 3, figure 4 and supplementary figures S1-S3); and 4) lower protein S levels in COPD patients with higher CAT score $(p=0.033)$ (table 3 and supplementary figure S2). Additional associations between coagulation factors and various parameters are presented in supplementary tables S2 and S3.

Association of coagulation factor levels with COPD parameters, clinical phenotypes and inflammatory markers

Univariate analyses of coagulation factors and inhibitors with various parameters are presented in supplementary tables S4-S11. After Bonferroni correction, parameters that remained significant were introduced in a multiple linear regression model and controlled for variables known to affect coagulation, including age, BMI, smoking status (pack-years and current smoking), CRP, neutrophil blood count and LDL serum levels. The following associations were significant: fibrinogen was positively associated with CRP $(p<0.0001)$ (see supplementary table S4); FII was negatively associated with $\mathrm{FEV}_{1}(\mathrm{p}=0.011)$ and age $(p=0.009)$ and positively associated with LDL $(p=0.035)$ (see supplementary table S5); FV was negatively associated with $\mathrm{FEV}_{1}(\mathrm{p}=0.003$ ) (see supplementary table S6); FVIII was positively associated with blood neutrophil count $(\mathrm{p}=0.015)$ (see supplementary table S7); FX was negatively associated with $\mathrm{FEV}_{1}(\mathrm{p}=0.002)$ and positively associated with LDL $(\mathrm{p}<0.001)$ (see supplementary table S8); D-dimers were positively associated with pack-years $(p=0.004)$ (see supplementary table $S 9$ ); protein $S$ was positively associated with CRP $(p=0.016)$ and negatively associated with age $(p=0.0001)$ (see supplementary table S10); antithrombin was negatively associated with blood neutrophil count $(\mathrm{p}<0.001)$, CRP $(p=0.038)$ and age $(p=0.001)$ (see supplementary table S11).

\section{Discussion}

In the present study, patients with stable COPD exhibited a prothrombotic state compared to COPD-free subjects with a history of smoking and equivalent burden of comorbidities, manifested by significantly higher levels of D-dimers, fibrinogen, FII, FV, FVIII and FX and significantly lower levels of coagulation inhibitors, namely protein S and antithrombin. Among patients with COPD, those with lower lung function 
TABLE 1 Baseline characteristics and comorbidities of patients with stable COPD and control subjects

\begin{tabular}{|c|c|c|c|}
\hline & Control group & COPD & p-value ${ }^{\#}$ \\
\hline Subjects $\mathrm{n}$ & 42 & 103 & \\
\hline \multicolumn{4}{|l|}{ Demographic factors } \\
\hline Age years & $62.52 \pm 8.76$ & $62.91 \pm 7.27$ & 0.638 \\
\hline Male sex $\mathrm{n}(\%)$ & $40(96)$ & $100(97)$ & 0.723 \\
\hline Current smokers n (\%) & $20(47.6)$ & $43(41.7)$ & 0.452 \\
\hline Pack-years & $30(20-48)$ & $60(50-80)$ & 0.001 \\
\hline $\mathrm{BMI}$ & $29.4(26.2-32.5)$ & $26.9(24.7-29.7)$ & 0.015 \\
\hline \multicolumn{4}{|l|}{ Spirometric parameters } \\
\hline $\mathrm{FEV}_{1} \%$ & $97.74 \pm 15.98$ & $55.80 \pm 16.85$ & $<0.001$ \\
\hline $\mathrm{FEV}_{1} \mathrm{~L}$ & $3.26(2.66-3.56)$ & $1.56(1.22-2.02)$ & $<0.001$ \\
\hline $\mathrm{FEV}_{1} / \mathrm{FVC} \%$ & $83(78-86)$ & $61(51-68)$ & 0.001 \\
\hline $\mathrm{RV}$ & $101 \pm 14$ & $114 \pm 25$ & $<0.001$ \\
\hline TLC & $93 \pm 10$ & $87 \pm 14$ & 0.007 \\
\hline DLCOSB & $84 \pm 10$ & $56 \pm 22$ & 0.001 \\
\hline \multicolumn{4}{|l|}{ Comorbidities } \\
\hline HEART score & $5(3-7)$ & $5(4-7)$ & 0.314 \\
\hline Cardiovascular risk & $5(4-5)$ & $5(4-5)$ & 0.253 \\
\hline Charlson comorbidity index & $3(2-3)$ & $3(2-3)$ & 0.129 \\
\hline Arterial hypertension $\mathrm{n}(\%)$ & $28(66.7)$ & $58(56.3)$ & 0.270 \\
\hline Diabetes $\mathrm{n}(\%)$ & $8(19)$ & $19(18.4)$ & 0.993 \\
\hline Dyslipidaemia n (\%) & $20(47.6)$ & $37(35.9)$ & 0.197 \\
\hline \multicolumn{4}{|l|}{ Laboratory biomarkers } \\
\hline Neutrophils/ $\mu \mathrm{L}$ & $4300(3235-5280)$ & $5040(4130-5940)$ & 0.018 \\
\hline $\mathrm{WBC} / \mu \mathrm{L}$ & $6970(6065-8760)$ & $8030(6590-9570)$ & 0.030 \\
\hline ESR $\mathrm{mm} \cdot \mathrm{h}^{-1}$ & $15(11-20)$ & $19(13-27)$ & 0.010 \\
\hline $\mathrm{CRP} \mathrm{mg} \cdot \mathrm{L}^{-1}$ & $4(2-5)$ & $4(3-8)$ & 0.128 \\
\hline Ferritin $\mathrm{ng} \cdot \mathrm{mL}^{-1}$ & $98(38-159)$ & $108(59-156)$ & 0.323 \\
\hline Uric acid $\mathrm{mg} \cdot \mathrm{dL}^{-1}$ & $6(5.1-7.2)$ & $5.9(5.2-6.8)$ & 0.752 \\
\hline $\mathrm{LDL} \mathrm{mg} \cdot \mathrm{dL}^{-1}$ & $115.1 \pm 31.5$ & $129.9 \pm 38.4$ & 0.022 \\
\hline Glucose $\mathrm{mg} \cdot \mathrm{dL}^{-1}$ & $98(88-108)$ & $100(91-113)$ & 0.162 \\
\hline HbAlc \% & $5.9(5.55-6.25)$ & $5.8(5.5-6.3)$ & 0.995 \\
\hline Insulin $\mathrm{mIU} \cdot \mathrm{L}^{-1}$ & $7.2(4.7-10.7)$ & $8(4.7-12)$ & 0.515 \\
\hline HOMA-IR & $1.6(1.2-3.2)$ & $1.9(1-3)$ & 0.972 \\
\hline
\end{tabular}

$\mathrm{n}=145$. All values are presented as mean $\pm \mathrm{SD}$, median (interquartile range) or $\mathrm{n}(\%)$. SD: standard deviation; BMI: body mass index; FEV ${ }_{1}$ : forced expiratory volume in $1 \mathrm{~s}$; FVC: forced vital capacity; RV: residual volume; TLC: total lung capacity; DLCOSB: diffusing capacity of lung for carbon monoxide; WBC: white blood cells; ESR: erythrocyte sedimentation rate; CRP: C-reactive protein; LDL: low-density lipoprotein cholesterol; HbA1c \%: glycated haemoglobin \%; HOMA-IR: homeostatic model assessment for insulin resistance. ": Mann-Whitney U-test.

and frequent exacerbations had significantly increased levels of FII, FV and FX; those with higher CAT score had significantly increased levels of FX and decreased levels of protein S. In univariate analyses, severity of COPD was associated with increased levels of FII, FV and FX; systemic inflammation, characterised by elevated blood neutrophil count and CRP, was associated with increased levels of fibrinogen and FVIII, and decreased protein S and antithrombin levels; elevated serum LDL was associated with increased levels of FII and FX. In multiple linear regression analyses that accounted for prothrombotic risk factors, including age [16], BMI [17], smoking [18], CRP [19], neutrophil blood count [20] and LDL serum levels [21], the strength of these associations did not change.

Our data are in agreement with those of previous studies which found that stable COPD patients, compared with controls, had higher levels of FII, FV, FVII, FVIII and FIX and lower free tissue factor pathway inhibitor [14], as well as increased levels of fibrinogen and D-dimers [22, 23]. To the best of our knowledge, our study is the first one to report significant differences in the level of several coagulation factors among COPD patients, driven by severity of COPD, as well as significantly lower levels of protein $\mathrm{S}$ and antithrombin in stable COPD patients compared to COPD-free smokers.

Our results differ from those of a previous study which reported no associations of severity of COPD or inflammatory markers with levels of FII, FV and FVIII, among stable COPD patients [14]. The 


\section{TABLE 2 Coagulation factor levels in patients with stable COPD and control subjects}

\begin{tabular}{|c|c|c|c|}
\hline Coagulation factor levels & Control group & COPD & p-value \\
\hline Subjects $n$ & 42 & 103 & \\
\hline Fibrinogen $\mathrm{mg} \cdot \mathrm{dL}^{-1}$ & $346 \pm 65$ & $399 \pm 82$ & $<0.001$ \\
\hline FII \% & $109 \pm 19$ & $122 \pm 22$ & 0.004 \\
\hline FV \% & $112 \pm 19$ & $131 \pm 25$ & 0.015 \\
\hline FVII \% & $108 \pm 19$ & $115 \pm 23$ & 0.113 \\
\hline FVIII \% & $122 \pm 20$ & $143 \pm 32$ & $<0.001$ \\
\hline FIX \% & $111(106-123)$ & $116(108-126)$ & 0.126 \\
\hline FX \% & $98(88-107)$ & $111(94-134)$ & 0.002 \\
\hline $\mathrm{D}$-dimers $\mathrm{ng} \cdot \mathrm{mL}^{-1}$ & $240(180-400)$ & $360(230-600)$ & 0.001 \\
\hline INR & $1.01(0.95-1.05)$ & $1.00(0.93-1.05)$ & 0.853 \\
\hline PT s & $14.1(13.1-14.5)$ & $13.7(12.8-14.4)$ & 0.184 \\
\hline aPTT s & $29.8 \pm 3.0$ & $28.2 \pm 3.4$ & 0.515 \\
\hline Free protein $\mathrm{S}$ antigen $\%$ & $116(98-121)$ & $95(85-105)$ & $<0.001$ \\
\hline Protein C \% & $116.2 \pm 17.1$ & $112.6 \pm 18.1$ & 0.150 \\
\hline Antithrombin \% & $102.3 \pm 13.2$ & $94.4 \pm 11.5$ & 0.001 \\
\hline
\end{tabular}

$\mathrm{n}=145$. All values are presented as mean \pm SD or median (interquartile range). SD: standard deviation; PT: prothrombin time; aPTT: activated partial thromboplastin time. ": Mann-Whitney U-test.

discrepancies between the findings of the two studies might be due to differences in the severity of COPD between the two study groups, given that in the study of UnDAs et al. [14] only 5\% of COPD patients were classified to GOLD stage IV.

In prior studies elevated levels of FII, FVIII, FIX and FXI have primarily been associated with an increased risk of VTE [24, 25], while elevated levels of fibrinogen, FV and FVII have primarily been associated with an increased risk of CVD [26, 27]. Underlying mechanisms leading to increased prevalence of cardiovascular and VTE events in COPD include platelet activation [10] and the presence of a prothrombotic state [11]. The prominent role of hypoxia and inflammation in the pathogenesis of VTE and CVD in COPD has been highlighted by several studies showing an increased prevalence of VTE and major cardiac events during COPD exacerbations [28]. Hypoxia, either sustained or intermittent, during exercise or sleep, can activate coagulation and promote inflammation. Thus, a 2-hour hypoxic challenge in
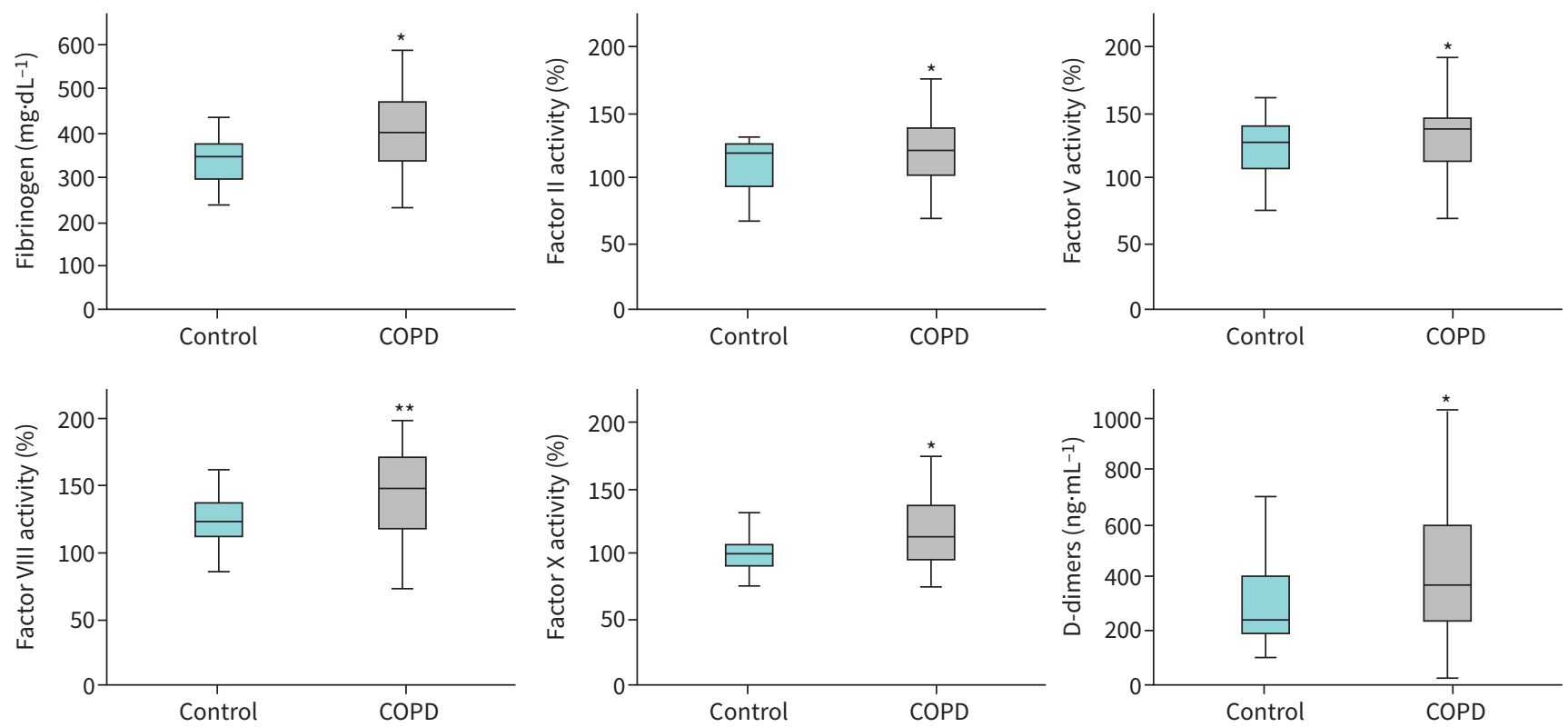

FIGURE 2 Levels of fibrinogen FII, FV, FVIII and FX, and D-dimers in control subjects ( $n=42$ ) and patients with stable COPD ( $n=103$ ). Boxplots display median with interquartile range and whiskers show minimum and maximum values $\left({ }^{*} p<0.05,{ }^{\star *} p<0.001\right.$, Mann-Whitney test). 

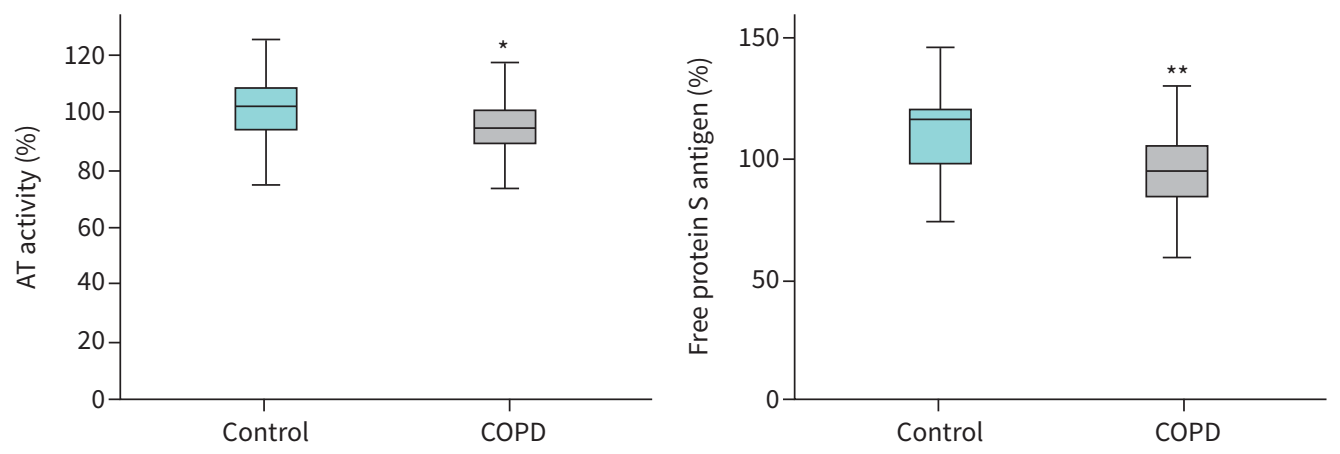

FIGURE 3 Levels of coagulation inhibitors antithrombin (AT) and protein $S$ in control subjects $(n=42)$ and patients with stable COPD $(n=103)$. Boxplots display median with interquartile range and whiskers show minimum and maximum values $\left({ }^{*} p<0.05,{ }^{\star *} p<0.001\right.$, Mann-Whitney test).

COPD patients led to increased thrombin-antithrombin complex, prothrombin activation fragments $1+2$ and interleukin-6 [29].

Neutrophilic inflammation and elevated CRP can also affect coagulation in COPD. Neutrophils play a significant role in thrombosis through their ability to release web-like structures composed of DNA filaments coated with histones and granule proteins referred to as neutrophil extracellular traps [20]. Neutrophilic inflammation is a prominent aspect of COPD, as neutrophils are the most abundant inflammatory cells present in the bronchial wall and lumen of COPD patients [30]. CRP has also been shown to be an activator of coagulation by inducing in vitro expression of tissue factor in peripheral blood monocytes [19], and by increasing blood levels of thrombin and plasminogen activator inhibitor Type-1 after infusion in healthy volunteers [31].

Systemic inflammation in COPD can be induced by COPD itself and comorbid conditions [32]. COPD patients suffer from a high number of comorbidities and up to two-thirds of individuals with COPD die of non-pulmonary causes [33, 34]. In the BODE COPD cohort comprising 1664 patients with COPD, 79 comorbidities were described [33]. Therefore, the prothrombotic state exhibited by COPD patients in this study might be linked to COPD itself and underlying multimorbidity.

TABLE 3 Associations of fibrinogen, FII, FV, FVIII, FX, protein S, antithrombin (AT) and D-dimers with spirometric stages, GOLD ABCD groups, CAT score and number of exacerbations in patients with stable COPD

\begin{tabular}{|c|c|c|c|c|c|c|c|c|c|}
\hline & n (\%) & I & II & v & VIII & $x$ & Protein S & AT & D-dimers \\
\hline \multicolumn{10}{|c|}{$\mathrm{FEV}_{1} \%$ predicted } \\
\hline$\geqslant 50 \%$ & $62(60.2)$ & $403 \pm 82$ & $117 \pm 17$ & $127 \pm 24$ & $144(110-156)$ & $102(86-118)$ & $95(83-107)$ & $94 \pm 11.7$ & $375(230-560$ \\
\hline$<50 \%$ & $41(39.2)$ & $391 \pm 81$ & $130 \pm 27$ & $138 \pm 24$ & $154(126-182)$ & $124(110-143)$ & $95(85-105)$ & $95 \pm 11.3$ & $350(200-630$ \\
\hline p-value ${ }^{\#}$ & & 0.574 & 0.003 & 0.018 & 0.729 & 0.001 & 0.656 & 0.813 & 0.696 \\
\hline \multicolumn{10}{|c|}{ GOLD groups } \\
\hline A & $28(27.2)$ & $407 \pm 84$ & $114 \pm 17$ & $123 \pm 22$ & $135 \pm 29$ & $94(88-113)$ & $95(88-112)$ & $94(89-100)$ & $340(252-470$ \\
\hline B & $27(26.2)$ & $397 \pm 82$ & $116 \pm 16$ & $133 \pm 27$ & $140 \pm 33$ & $122(95-137)$ & $91(76-104)$ & $94(89-97)$ & $450(240-750$ \\
\hline C & $15(14.6)$ & $420 \pm 75$ & $129 \pm 22$ & $133 \pm 22$ & $145 \pm 28$ & $128(108-140)$ & 98 (95-109) & $96(94-101)$ & $330(220-780$ \\
\hline D & $33(32)$ & $383 \pm 83$ & $130 \pm 27$ & $135 \pm 26$ & $150 \pm 33$ & $114(102-139)$ & $95(80-105)$ & $94(85-103)$ & $360(225-630$ \\
\hline p-value & & 0.482 & 0.017 & 0.147 & 0.180 & 0.002 & 0.177 & 0.770 & 0.529 \\
\hline \multicolumn{10}{|l|}{ CAT score } \\
\hline$<10$ & $43(41.7)$ & $389 \pm 82$ & $120 \pm 20$ & $127 \pm 22$ & $140(117-159)$ & $101(90-128)$ & 96 (92-109) & $94(89-101)$ & $330(250-530$ \\
\hline$\geqslant 10$ & $60(58.3)$ & $411 \pm 80$ & $124 \pm 24$ & $134 \pm 26$ & 153(117-170) & $118(100-138)$ & $94.5(77-104)$ & $94(87-100)$ & $420(230-655$ \\
\hline$p$-value ${ }^{\#}$ & & 0.199 & 0.418 & 0.066 & 0.167 & 0.034 & 0.033 & 0.608 & 0.304 \\
\hline \multicolumn{10}{|c|}{ Exacerbations } \\
\hline$<2$ & $78(75.7)$ & $399 \pm 81$ & $114 \pm 16$ & $124 \pm 23$ & $144(117-167)$ & 99 (89-123) & $95 \pm 19$ & $94 \pm 12$ & $380(248-618$ \\
\hline$\geqslant 2$ & $25(24.3)$ & $397 \pm 85$ & $127 \pm 24$ & $136 \pm 25$ & $158(120-170)$ & 121 (101-139) & $94 \pm 17$ & $96 \pm 11$ & $340(225-535$ \\
\hline p-value & & 0.866 & 0.005 & 0.018 & 0.368 & 0.003 & 0.714 & 0.512 & 0.593 \\
\hline
\end{tabular}



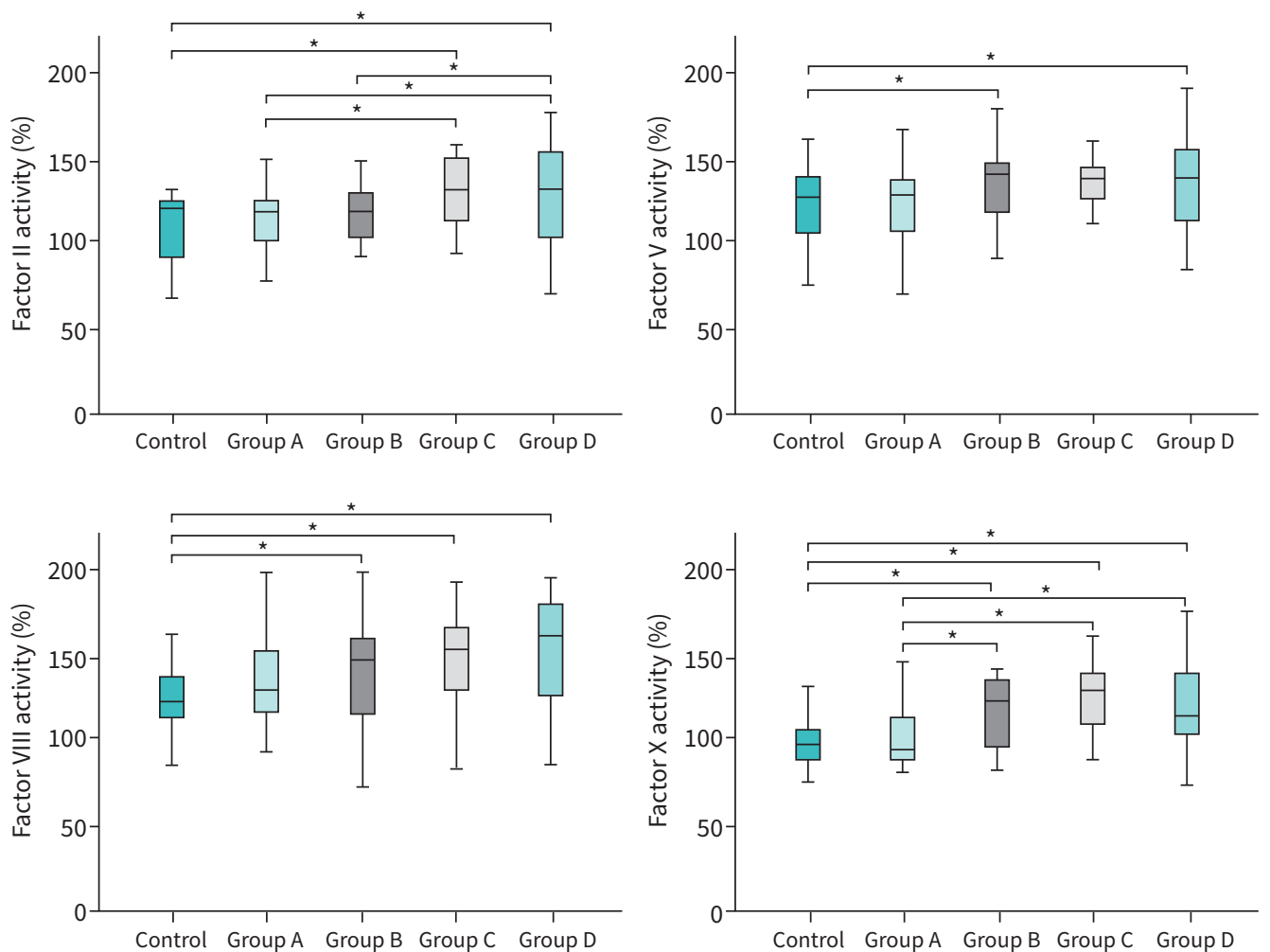

FIGURE 4 Levels of coagulation factors II, V, VIII and X in control subjects ( $n=42)$ and Groups A-D of patients with stable COPD ( $n=103$ ), classified according to GOLD COPD 2021 guidelines ( ${ }^{*} p<0.05$, Kruskal-Wallis test). Group A, n=28; Group B, n=27; Group C, n=15; Group D, n=33.

Levels of FII and FX were positively associated with LDL levels in our study. High levels of blood lipids have been associated with high levels of coagulation factors, including significant correlations of FII and FX with total cholesterol [21]. Furthermore, hyperlipidaemia has a very high prevalence in COPD, affecting up to $50 \%$ of the patients [33]. Diabetes can induce a prothrombotic state [35]; therefore, we performed a separate analysis of study participants excluding those with diabetes. We found the same coagulation factors being significantly different between the two groups, providing evidence that diabetes was not a contributor to the prothrombotic state in our study.

A crucial question is whether the relatively small, but significant, magnitude of increase in coagulation factors and decrease in coagulation inhibitors in patients with COPD in our study can indeed induce a prothrombotic state and be clinically relevant. Data from previous studies provide evidence that the thrombotic risk conferred by increased levels of FVIII, fibrinogen, D-dimers and FV is not defined by a critical cut-off value; instead, all of them are dose-dependent thrombotic risk factors. In the Leiden Thrombophilia Study, the risk of VTE increased with a small increase of FVIII levels. Thus, the odds ratio for DVT was 2.3 for FVIII activity levels between 100 and $125 \mathrm{IU} \cdot \mathrm{dL}$ versus $<100 \mathrm{IU} \cdot \mathrm{dL}, 3$ for levels between 125 and $150 \mathrm{IU} \cdot \mathrm{dL}$ and 4.8 for levels $>150 \mathrm{IU} \cdot \mathrm{dL}$ [24]. Similarly, in a large meta-analysis, an approximately log-linear association between fibrinogen level and the risk for CVD and stroke with hazard ratios of about 1.8 per $1 \mathrm{~g} \cdot \mathrm{L}^{-1}$ increase in usual fibrinogen level was demonstrated [36]. In another study of patients with stable coronary heart disease with a median follow-up of 6 years, risks of major cardiovascular events increased with increasing D-dimer levels with adjusted hazard ratios of 1.44, 1.45 and 4.03 for patients in the highest quartile in comparison with patients in the lowest quartile, with most patients having D-dimer levels well below $500 \mathrm{ng} \cdot \mathrm{mL}^{-1}$, which is the cut-off used for the diagnosis of acute VTE [37]. Furthermore, in a case-control study analysing the relative risk for myocardial infarction associated with increased FV activity, subjects in the highest quartile, defined by FV activity of $>106 \%$, were found to have a 3.3-fold risk compared to those in the first quartile (those with FV activity <86\%) [27]. Finally, although VTE risk in antithrombin deficiency is usually defined by antithrombin levels $<70 \%$, in a population-based cohort study composed of patients with a first VTE, an increased risk of recurrent VTE in those with antithrombin activity $<87 \%$ was found (hazard ratio 1.5 ), providing evidence that even mild antithrombin deficiency predisposes to recurrent VTE [38]. 
The main strengths of the present study were the presence of similar comorbidity burden and cardiovascular risk in both patients with stable COPD and COPD-free smokers, the assessment of key coagulation factors and coagulation inhibitors in both groups and the analyses performed in the group of patients with COPD.

Our study has several limitations. First, to avoid recruitment of subjects suffering from certain comorbidities that are associated with a hypercoagulable state, we have excluded both COPD patients and control subjects with those comorbidities using the same exclusion criteria. Although this approach has minimised the effect of confounding variables, it has led to a considerable reduction in the number of participants, as well as an imbalance in the number of patients in each COPD group, thus limiting the generalisability of our findings. This also led to the exclusion of patients with severe comorbidities encountered in COPD, such as coronary artery disease, atrial fibrillation and renal disease. However, we can speculate that most patients with comorbidities and COPD may exhibit even more severe prothrombotic alterations. In addition, patients recruited exhibited comorbidities frequently associated with COPD, including arterial hypertension, dyslipidaemia and Type 2 diabetes. Because of several exclusion criteria, during the design of our study we decided to recruit well-characterised control subjects from the same outpatient respiratory clinic rather than recruiting them from the community, as the thorough evaluation and the available medical information would allow optimal recruitment. Second, we recruited control subjects of similar sex and age, but we were unable to recruit control subjects with similar smoking history, given that patients with COPD were heavy smokers. Therefore, we cannot exclude the possibility that the discordance in smoking history (higher in the COPD group) may have played a role in altered levels of coagulation factors and inhibitors, since smoking can induce a hypercoagulable state [39, 40]. Nevertheless, matching for smoking history represents an inherent challenge in studies involving COPD patients. Third, there are additional confounders that can potentially affect thrombogenesis that we were unable to adjust for in the present study, including lifestyle habits such as physical activity and alcohol intake [41]. Fourth, we have not analysed hypoxia as an independent factor of prothrombotic state because we have not collected data on the oxygenation status, such as arterial blood gases or overnight oximetry, from all patients and control subjects. Finally, most of our patients were male and a significant number of patients with mild/moderate COPD were included. Since smoking has been more prevalent among men in Greece, COPD patients followed at our outpatient respiratory clinic have been predominantly males. The relatively high number of group A patients in this study is likely due to the fact that our outpatient respiratory clinic, where patients and controls were recruited, does not only serve as a dedicated referral clinic for severe COPD patients by primary care physicians, but also allows patients to book an appointment on their own.

\section{Conclusion}

In conclusion, this study showed that stable COPD patients exhibit a prothrombotic state characterised by increased levels of D-dimers, fibrinogen, FII, FV, FVIII and FX, and decreased levels of coagulation inhibitors, namely protein $\mathrm{S}$ and antithrombin, compared to COPD-free smokers with similar comorbidity burden and cardiovascular risk. Among patients with stable COPD, levels of FII, FV, FX, protein S and D-dimers were associated with COPD severity; levels of fibrinogen, FVIII, protein S and antithrombin with CRP and neutrophil blood count; and levels of FII and FX with serum LDL. Although the above associations are not causative, they generate new hypotheses that require further evaluation in cohort studies with a larger sample size.

Acknowledgements: We thank the study participants, and their families and guardians, for their participation in this study.

Provenance: Submitted article, peer reviewed.

Author contributions: C. Kyriakopoulos is the first author; wrote the first manuscript draft together with A. Konstantinidis; was responsible for study conception and design; and performed the statistical analysis with input from A.Tatsioni, A. Konstantinidis and K. Kostikas. C. Chronis was responsible for study conception and design; and recruited and followed up patients. E. Papetrou was responsible for study conception and design, and blood collection and laboratory analysis. A. Tatsioni contributed to the statistical analysis and was involved with interpretation of data. K. Gartzonika contributed to the analysis and interpretation of data. C. Tsaousi contributed to blood collection and laboratory analysis. A. Gogali recruited and followed up patients, and contributed to data analysis. C. Katsanos recruited and followed up patients, and contributed to data analysis. A. Vaggeli recruited and followed up patients, and contributed to data analysis. C. Tselepi recruited patients and contributed to the data analysis. G. Daskalopoulos contributed to the analysis and interpretation of data. S. Constantopoulos contributed 
to the analysis and interpretation of data. K. Kostikas contributed to the statistical analysis and interpretation of data. A. Konstantinidis wrote the first manuscript draft together with C. Kyriakopoulos; was responsible for study conception and design, and contributed to the statistical analysis. All authors were involved in the interpretation of the data and the writing and critical review of the manuscript. All authors approved the final version before submission.

Conflict of interest: None declared.

\section{References}

1 Adeloye D, Chua S, Lee C, et al. Global and regional estimates of COPD prevalence: systematic review and meta-analysis. J Glob Health 2015; 5: 020415.

2 World Health Organization. 2020. www.who.int/news-room/fact-sheets/detail/the-top-10-causes-of-death Date last accessed: 15 July 2021.

3 Chen W, Thomas J, Sadatsafavi M, et al. Risk of cardiovascular comorbidity in patients with chronic obstructive pulmonary disease: a systematic review and meta-analysis. Lancet Respir Med 2015; 3: 631-639.

4 Børvik T, Brækkan SK, Enga K, et al. COPD and risk of venous thromboembolism and mortality in a general population. Eur Respir J 2016; 47: 473-481.

5 Schneider C, Bothner U, Jick SS, et al. Chronic obstructive pulmonary disease and the risk of cardiovascular diseases. Eur J Epidemiol 2010; 25: 253-260.

6 Couturaud F, Bertoletti L, Pastre J, et al. Prevalence of pulmonary embolism among patients with COPD hospitalized with acutely worsening respiratory symptoms. JAMA 2021; 325: 59-68.

7 Polosa R, Malerba M, Cacciola RR, et al. Effect of acute exacerbations on circulating endothelial, clotting and fibrinolytic markers in COPD patients. Intern Emerg Med 2013; 8: 567-574.

8 Lankeit M, Held M. Incidence of venous thromboembolism in COPD: linking inflammation and thrombosis? Eur Respir J 2016; 47: 369-373.

9 Sin DD, Wu L, Man SF. The relationship between reduced lung function and cardiovascular mortality. Chest 2005; 127: 1952-1959.

10 Fimognari FL, Scarlata S, Conte ME, et al. Mechanisms of atherothrombosis in chronic obstructive pulmonary disease. Int J Chron Obstruct Pulmon Dis 2008; 3: 89-96.

11 Fimognari FL, Scarlata S, Antonelli-Incalzi R. Why are people with "poor lung function" at increased atherothrombotic risk? A critical review with potential therapeutic indications. Curr Vasc Pharmacol 2010; 8: 573-586.

12 Vaidyula VR, Criner GJ, Grabianowski C, et al. Circulating tissue factor procoagulant activity is elevated in stable moderate to severe chronic obstructive pulmonary disease. Thromb Res 2009; 124: 259-261.

13 Ashitani J, Mukae $\mathrm{H}$, Arimura $\mathrm{Y}$, et al. Elevated plasma procoagulant and fibrinolytic markers in patients with chronic obstructive pulmonary disease. Intern Med 2002; 41: 181-185.

14 Undas A, Jankowski M, Kaczmarek P, et al. Thrombin generation in chronic obstructive pulmonary disease: dependence on plasma factor composition. Thromb Res 2011; 128: e24-e28.

15 von Elm E, Altman DG, Egger M, et al. The Strengthening the Reporting of Observational Studies in Epidemiology (STROBE) statement: guidelines for reporting observational studies. J Clin Epidemiol 2008; 61: 344-349.

16 Mari D, Ogliari G, Castaldi D, et al. Hemostasis and ageing. Immun Ageing 2008; 5: 12.

17 Samad F, Ruf W. Inflammation, obesity, and thrombosis. Blood 2013; 122: 3415-3422.

18 Barua RS, Ambrose JA, Saha DC, et al. Smoking is associated with altered endothelial-derived fibrinolytic and antithrombotic factors: an in vitro demonstration. Circulation 2002; 106: 905-918.

19 Bisoendial RJ, Kastelein JJ, Levels JH, et al. Activation of inflammation and coagulation after infusion of C-reactive protein in humans. Circ Res 2005; 96: 714-716.

20 Noubouossie DF, Reeves BN, Strahl BD, et al. Neutrophils: back in the thrombosis spotlight. Blood 2019; 133: 2186-2197.

21 Kim J-A, Kim J-E, Song SH, et al. Influence of blood lipids on global coagulation test results. Ann Lab Med 2015; 35: 15-21.

22 Duvoix A, Dickens J, Haq I, et al. Blood fibrinogen as a biomarker of chronic obstructive pulmonary disease. Thorax 2013; 68: 670-676.

23 Silva DR, Coelho AC, Gazzana MB, et al. D-dimer levels in stable COPD patients: a case-control study. COPD 2012; 9: 426-431.

24 van der Meer FJ, Koster T, Vandenbroucke JP, et al. The Leiden Thrombophilia Study (LETS). Thromb Headmost 1997; 78: 631-635.

25 van Hylckama Vlieg A, van der Linden IK, Bertina RM, et al. High levels of factor IX increase the risk of venous thrombosis. Blood 2000; 95: 3678-3682.

26 Chandler WL, Rodgers GM, Sprouse JT, et al. Elevated hemostatic factor levels as potential risk factors for thrombosis. Arch Pathol Lab Med 2002; 126: 1405-1414. 
Redondo M, Watzke HH, Stucki B, et al. Coagulation factors II, V, VII, and X, prothrombin gene 20210G-->A transition, and factor $\mathrm{V}$ Leiden in coronary artery disease: high factor $\mathrm{V}$ clotting activity is an independent risk factor for myocardial infarction. Arterioscler Thromb Vasc Biol 1999; 19: 1020-1025.

28 Gunen H, Gulbas G, In E, et al. Venous thromboemboli and exacerbations of COPD. Eur Respir J 2010; 35: 1243-1248.

29 Sabit R, Thomas P, Shale DJ, et al. The effects of hypoxia on markers of coagulation and systemic inflammation in patients with COPD. Chest 2010; 138: 47-51.

30 Hoenderdos K, Condliffe A. The neutrophil in chronic obstructive pulmonary disease. Am J Respir Cell Mol Biol 2013; 48: 531-539.

31 Cermak J, Key NS, Bach RR, et al. C-reactive protein induces human peripheral blood monocytes to synthesize tissue factor. Blood 1993; 82: 513-520.

32 Agustí A, Faner R. Systemic inflammation and comorbidities in chronic obstructive pulmonary disease. Proc Am Thorac Soc 2012; 9: 43-46.

33 Divo MJ, Casanova C, Marin JM, et al. COPD comorbidities network. Eur Respir J 2015; 46: 640-650.

34 Divo M, Celli BR. Multimorbidity in patients with chronic obstructive pulmonary disease. Clin Chest Med 2020; 41: 405-419.

35 van der Toorn FA, de Mutsert R, Lijfering WM, et al. Glucose metabolism affects coagulation factors: the NEO study. J Thromb Haemost 2019; 17: 1886-1897.

36 Fibrinogen Studies Collaboration, Danesh J, Lewington S, et al. Plasma fibrinogen level and the risk of major cardiovascular diseases and nonvascular mortality: an individual participant meta-analysis. JAMA 2005; 294: 1799-1809.

37 Simes J, Robledo KP, White HD, et al. D-dimer predicts long-term cause-specific mortality, cardiovascular events, and cancer in patients with stable coronary heart disease: LIPID study. Circulation 2018; 138: 712-723.

38 Sokol J, Timp JF, le Cessie S, et al. Mild antithrombin deficiency and risk of recurrent venous thromboembolism: results from the MEGA follow-up study. J Thromb Haemost 2018; 16: 680-688.

39 Sambola A, Osende J, Hathcock J, et al. Role of risk factors in the modulation of tissue factor activity and blood thrombogenicity. Circulation 2003; 107: 973-977.

40 Ariëns RA, Kohler HP, Mansfield MW, et al. Subunit antigen and activity levels of blood coagulation factor XIII in healthy individuals. Relation to sex, age, smoking, and hypertension. Arterioscler Thromb Vasc Biol 1999; 19: 2012-2016.

41 Lee KW, Lip GY. Effects of lifestyle on hemostasis, fibrinolysis, and platelet reactivity: a systematic review. Arch Intern Med 2003; 163: 2368-2392. 